\title{
CARACTERIZAÇÃo PRELIMINAR DE Amaranthus hybridus L. E Amaranthus viridis I. ATRAVÉS DE ISOENZIMAS
}

ANGELA MARIA MALUF ${ }^{1}$ PAULO SODERO MARTINS ${ }^{2}$

RESUMO: Através da técnica de eletroforese de gel de amido com migração horizontal, pode-se observar que para esterase, apenas o Amaranthus hybridus tipo verde apresentou variação, na forma de presença ou ausência da banda na posiÇão 0.7. Por outro lado, para peroxidase, para todas as espécies e biótipos (A.viridis e A.hybridus tipo verde e tipo roxol houve variação no padrão de bandas. Através da existência de bandas comuns às duas espécies, pode-se detectar evidências de hibridação e introgressão entre elas.

Termos para Indexação: eletroforese, isoenzima, esterase, peroxidase, Amaranthus, hibridação, introgressão

PRELIMINARY CHARACTERIZATION OF AmaranthuS hybridus L. AND Amaranthus viridis L. THROUGH ISOZYMES

ABSTRACT - Through starch gel electrophoresis with horizontal migration, Amaranthus hybridus green biotype was the only accession to show variation in esterase patterns, with presence or absence of a band in position 0.7 . On the other hand, all species and biotypes (A.viridis

1 Instituto de Botânica, Caixa Postal, 4005, São Paulo-SP - CEP 01015

2 Departamento de Genética, ESALQ/USP, Caixa Postal. 83 - PIRACICABA - SP - CEP 13400 
and A.hybridus green and purple biotypes) showed variation in banding patterns for peroxidase. Through the existence of common bands to both species, one could detect evidence for hybridization and introgression between them. Index Terms:
electrophoresis,
isozyme,

esterase, hybridization, introgression.

\section{INTRODUÇĀO}

A presença de variabilidade genética dentro da espécie é que torna possivel sua adaptação às mudanças ambientais e permite sua evolução. Esta variabilidade, existente em populaçōes naturais, vem sendo atualmente estudada através da extração e análise da mobilidade de proteínas solúveis, particularmente enzimas. Além disso, tem-se dado ênfase à identificação de espécies, variedades e cultivares de plantas através da análise enzimática usando a técnica de eletroforese (DAMANIA et alii, 1983; COLLINS et alii, 1984; ATKINSON et alii, 1986; HUSSAIN et alii, 1986 e 1987). Temse também procurado relacionar padrões de variação eletroforética com variação morfológica da planta (MARTINS \& JAIN, 1980; RITLAND \& JAIN, 1984; SCHWAEGERLE et alii, 1986; MARCON, 1988 ).

Em Amaranthus, alguns trabalhos foram realizados usando a eletroforese para o estudo do polimorfismo em diversas populações e espécies. JAIN et alii (1980) observaram altos niveis de polimorfismo morfológico nos Amaranthus cultivados da India, tanto do tipo de folhas como de grãos, contrastando com locos a loz imaticos monomórficos. Encontraram polimorfismo apenas para os locos de esterase, enquanto que os locos para álcool desidrogenase $(A D H)$, leucina aminopeptidase (LAP), fosfatase ácida $(\mathrm{ACPH})$ e glutamato oxaloacetatc " "sami- 
nase (GOT) mostraram-se monomórficos. No entanto, os alelos alozimáticos fixados distinguiram consistentemente os tipos de folhas dos de grãos.

Através da análise de nove sistemas enzimáticos, HAUPTLI \& JAIN (1984) verificaram que três espécies domesticadas de Amaranthus (A.caudatus, A.quitensis e A.retroflexus) estão geneticamente mais próximos entre si do que com relação aos seus pretensos progenitores com hábito colonizador. Isto parece indicar um único evento de domesticação envolvendo A.hybridus como ancestral comum, e não três eventos separados envolvendo cada uma das espécies. Observaram também, algumas evidências de introgressão recente e/ou segregação de híbridos de espécies cultivadas e colonizadoras entre A.caudatus e A.retroflexus. As populaçōes dos amarantos graníferos do centro de origem do Novo Mundo apresentaram padrões de variação isoenzimática que sugerem que, a maior parte das variedades locais é uma mistura de genótipos altamente homozigotos (HAUPTLI \& JAIN, 1983).

ZANGERL \& BAZZAZ (1984a), encontraram respostas diferenciais na germinação de sementes de genótipos de A.retroflexus homogêneos para o loco da fosfoglucoisomerase (PGI). Quando estes genótipos foram submetidos a quatro ciclos de seleção sob diversos tratamentos de estresse ambiental (densidade de plantas, densidade de competidores e umidade do solo) ZANGERL \& BAZZAZ (1984a) verificaram que, apesar da divergência destas populaçōes experimentais com o estoque original, em uma ou mais características fisiológicas, morfológicas ou eletroforéticas, foi encontrada pequena divergência no comportamento ecológico.

o presente trabalho teve por objetivo determinar os padrões de migração isoenzimática, para esterase e peroxidase, em Amaranthus viridis L. e em dois biótipos de Amaranthus 
hybridus L. (tipo verde e tipo roxo), para detectar evidência de cruzamento entre as espécies.

\section{MATERIAL E MÉTODOB}

o trabalho foi realizado no Laboratório de Genética Ecológica do Departamento de Genética da ESALQ/USP, em Piracicaba.

Foram utilizadas duas espécies de Amaranthus L., A. viridis L. e A. hybridus L., sendo que esta última espécie apresentava dois biótipos distintos (planta verde e planta ro$x a)$. As coletas foram realizadas em cinco locais, sendo que no local 1, duas espécies estavam associadas (A.viridis e A.hybridus tipo verde); no local 2 , ocorria A.viridis e os dois biotipos de $A$. hybridus (tipo verde e tipo roxo) e nos locais 3,4 e 5, ocorria apenas o biotipo verde de A.hybridus. As espécies, 10cais de coleta, época de coleta de sementes e numero de individuos coletados e amostrados encontram-se na Tabela 1 .

Para identificar os materiais no decorrer do trabalho, cada um deles recebeu uma sigla com duas letras (iniciais da espécie) e um número que se refere ao local da coleta. No caso da espécie de A.hybridus, a sigla contém ainda a letra $v$ ou $r$, indicando o biótipo verde ou roxo, respectivamente.

o número de individuos amostrados foi inferior ao número de individuos coletados devido à ocorrência de dormência nas sementes, - que provocou uma baixa germinação das mesmas.

Foi utilizada a técnica de eletroforese de gel de amido com migração horizontal, para detectar o nivel de variação isoenzimica existente entre os indivíduos das oito populaçōes de Amaranthus, descritas na Tabela 1, para dois sistemas enzimaticos, esterase e peroxidase. Testes preliminares indicaram a 


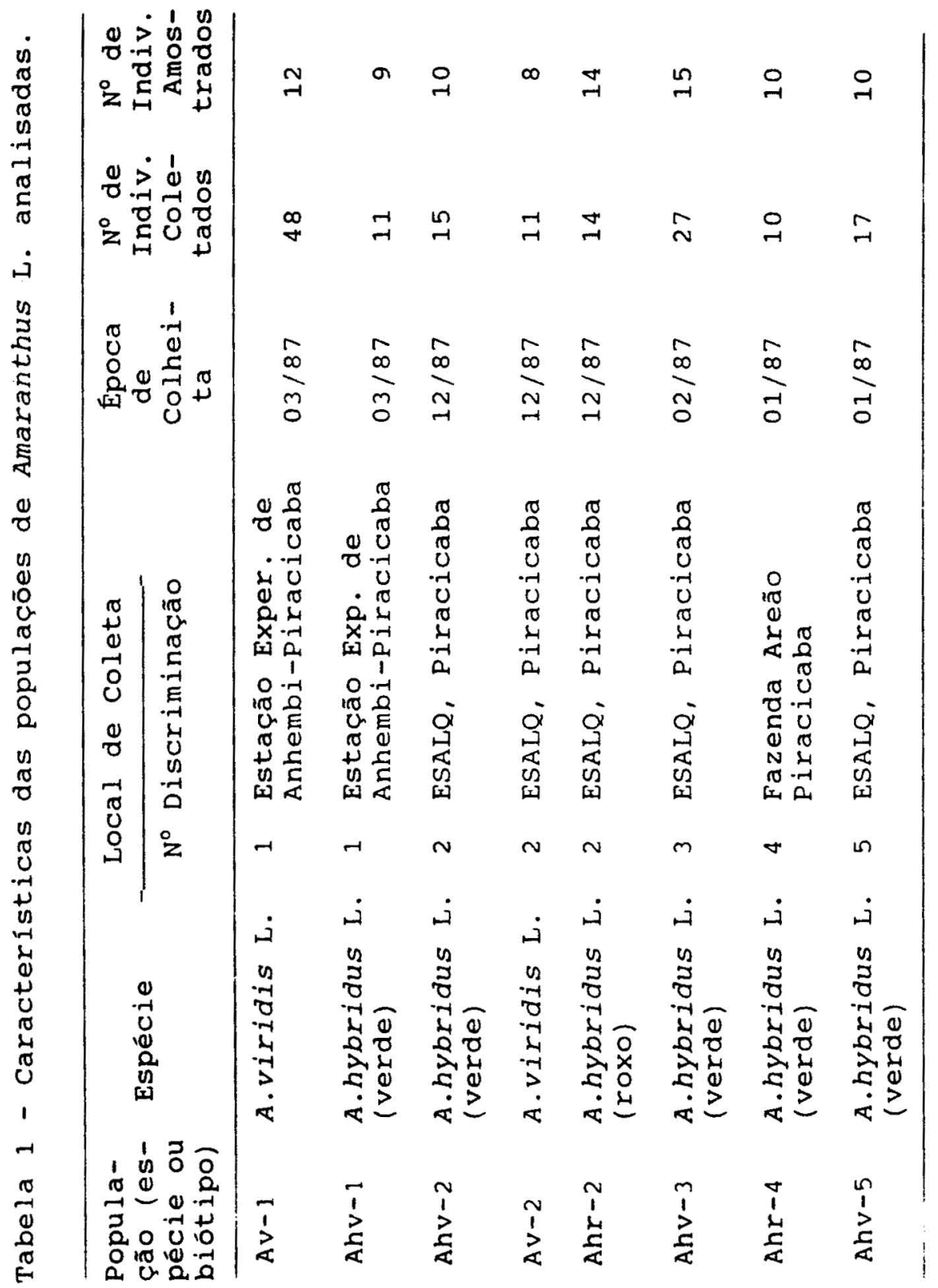


inexistência de atividade enzimática para catalase e malato desidrogenase nos materiais estudados.

Para a confecção do gel, de espessura de $2 \mathrm{~mm}$ e concentração de $12 \%$, utilizaram-se as soluções tampōes A e B de SCANDALIOS (1969), na proporção de 1A:9B e pH 8.3. Utilizaram-se dois tipos de amido: $2 / 3$ de amido nacional das Refinaçōes de Milho Brasil (Penetrose 30) e 1/3 de amido importado da marca Schiapparelli.

o gel foi feito com $125 \mathrm{ml}$ da solução tampão, $10 \mathrm{~g}$ de amido nacional e $5 \mathrm{~g}$ de amido importado. Foi resfriado à temperatura ambiente, sendo posteriormente resfriado a $4^{\circ} \mathrm{C}$, para receber os extratos das amostras homogeneizadas.

À semelhança do trabalho de HAUPTLI \& JAIN (1984), utilizaram-se plântulas com as duas primeiras folhas verdadeiras abertas para extração. As sementes individuais de cada população (Tabela 1) foram colocadas para germinar em estufas incubadoras para BOD, a $30^{\circ} \mathrm{C}$, na presença de luz, sobre o papel de filtro umidecido com água destilada.

os extratos de cada uma destas plântulas foram obtidos por maceração em $10 \mathrm{ml}$ de solução tampão do gel. Todas as etapas do processo, desde a homogeneização até a revelação das enzimas foram feitas em ambiente ou anteparo de contato frio $\left(4^{\circ} \mathrm{C}\right.$ a $\left.8^{\circ} \mathrm{C}\right)$.

Cada extrato foi absorvido em dois papéis filtro com dimensōes de $2 \mathrm{~mm} \times 1,5 \mathrm{~mm}$, um para ser usado para revelação da esterase e outro para peroxidase.

A aplicação das amostras no gel foi feita cortando-o com um pente de 25 dentes. Nos orificios, eram inseridos os papéis embebidos das amostras.

Para as peroxidases, as amostras foram aplicadas a $5 \mathrm{~cm}$ da ponte do polo $(-)$ e para as esterases, a $3 \mathrm{~cm}$ da ponte do polo (-), 
pois as duas enzimas apresentaram bandas anódicas.

Nas extremidades das aplicações, no $1^{\circ}$ e $25^{\circ}$ dentes do pente, foi colocada uma gota de azul de bromofenol para marcar a linha de frente da migração, o que permitiu saber quando interromper a corrida eletroforética e calcular a migração relativa (Rm) .

A migração (corrida eletroforética) foi realizada em geladeira, a $4{ }^{\circ} \mathrm{C}$, em cubas horizontais próprias. A solução tampão das cubas com os eletródios foi a solução tampão $A$ de SCANDALIOS (1969) com pH 8.3. Foi mantida uma distância uniforme de $12 \mathrm{~cm}$ entre as pontes catódica e anódica. A corrida eletroforética foi feita com uma diferença de potencial de $1 \mathrm{~mA} / \mathrm{cm}$ e $10 \mathrm{v} / \mathrm{cm}$ em corrente contínua. A migração foi interrompida quando a linha de frente atingiu $7 \mathrm{~cm}$ para peroxidase e $9 \mathrm{~cm}$ para esterase; isto aconteceu cerca de 14 horas após o início da corrida.

Após a corrida eletroforética foi feita a revelação, ou seja, a coloração do gel, para possibilitar a análise através dos zimogramas.

- sistema das peroxidases foi revelado pela técnica de BREWBAKER et alii (1968), modificada: etanol 958 ( $35 \mathrm{ml})$, solução tampão fosfato (14 ml), O-dianisidina (100 g) e $\mathrm{H}_{2} \mathrm{O}_{2} 1 \%$ (1 ml). A solução tampão fosfato (pH $6-1 \mathrm{M}$ ) foi constituida por $\mathrm{KH}_{2} \mathrm{PO}_{4}(109,0 \mathrm{~g}), \mathrm{K}_{2} \mathrm{HPO}_{4}$ $(34,8 \mathrm{~g})$ e $\mathrm{H}_{2} \mathrm{O}$ (até $1000^{2} \mathrm{ml}$ ). $\mathrm{O}$ pH 6 era ajustado com NaOH.

No sistema das esterases a revelação foi feita pela técnica da scANDALIOS (1969) modificada: $\mathrm{H}_{2} \mathrm{O}(20 \mathrm{ml})$, solução tampão $\mathrm{C}$ ( 25 ml), solução tampão D ( $5 \mathrm{ml})$, Fast Blue RR (sal) (40 mg) e $\alpha$-naftil acetato ( $1 \mathrm{ml}$ ).

A solução tampão $C$ : fosfato $(\mathrm{pH} 4.3$ $0,2 \mathrm{M})$ era constituía por $\mathrm{NaH}_{2} \mathrm{PO}_{4}(27,8 \mathrm{~g})$ e $\mathrm{H}_{2} \mathrm{O}$ (até $1000 \mathrm{ml}$ ). A solução tampão $\mathrm{D}$ : fosfato 
( $\mathrm{pH} 9,2-0,2 \mathrm{M}$ ) era constituído por $\mathrm{Na}_{2} \mathrm{PO}_{4}$ $(53,63 \mathrm{~g})$ e $\mathrm{H}_{2} \mathrm{O}$ (até $1000 \mathrm{ml}$ ).

o $\alpha$-naftil acetato foi dissolvido em etanol e água na proporção de $1 \mathrm{~g}$ de sal, $50 \mathrm{ml}$ de etanol e $50 \mathrm{ml}$ de água.

Os géis foram deixados em solução reveladora por uma hora, em temperatura ambiente, no escuro.

Após a revelação, os géis foram lavados e fixados em solução de água, álcool metilico e ácido acético na proporção de 5:5:1, por cerca de 15 minutos. Em seguida, foram lavados em água destilada, envoltos em plásticos, identificados e guardados em geladeira por 24 horas antes de serem analisados.

A leitura do gel foi esquematizada em papel milimetrado, anotando as bandas pela sua migração relativa $(\mathrm{Rm})$. O $\mathrm{Rm}$ de cada banda foi obtido pela divisão da distância migrada por ela, pela distância migrada pela linha de frente (azul de bromofenol).

o $\mathrm{Rm}$ permite determinar a homologia das bandas presentes em individuos diferentes ou diferentes tecidos de um mesmo organismo, ou em diferentes condições ambientais, populações ou periodos em que é feita a análise.

\section{RESULTADOS E DISCUSSĀO}

Os padrões de migração relativa (Rm) para as enzimas esterase $\left(E_{1}, E_{2}, E_{3} e E_{4}\right)$ e peroxidase $\left(P_{1}, P_{2}, P_{3}, P_{4}, P_{5}, P_{6}, P_{7}, P_{8}, e P_{9}\right)$ para Amaranthus hybridus tipo verde e tipo roxo e A.viridis podem ser vistos nas Figuras 1 e 2, respectivamente.

A Tabela 2 mostra a frequência, em porcentagem de ocorrência, dos padrōes de migração relativa entre os indivíduos de todas as populações utilizadas, para a enzima esterase. A Tabela 3 apresenta esta mesma frequência para a enzima peroxidase. 


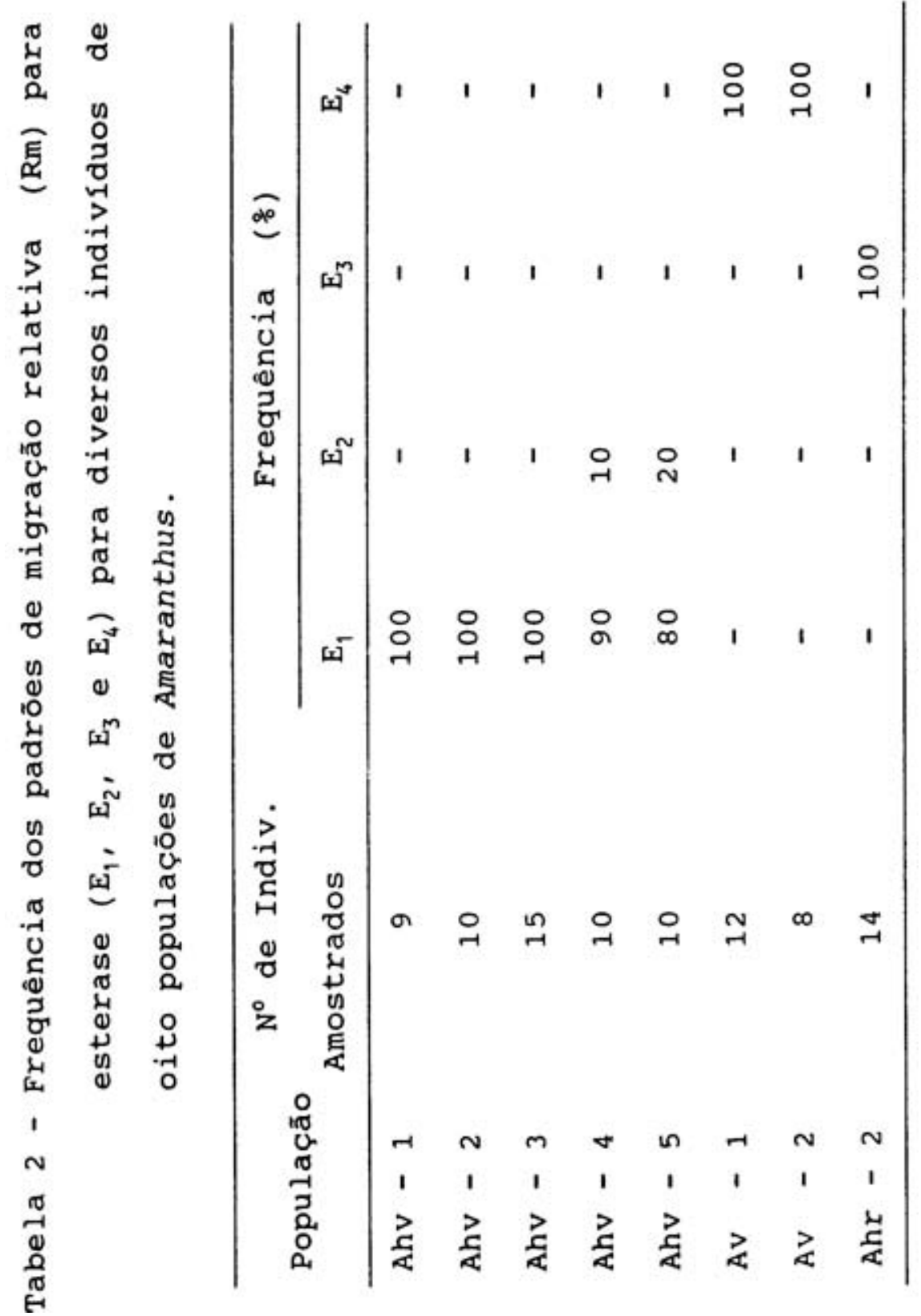




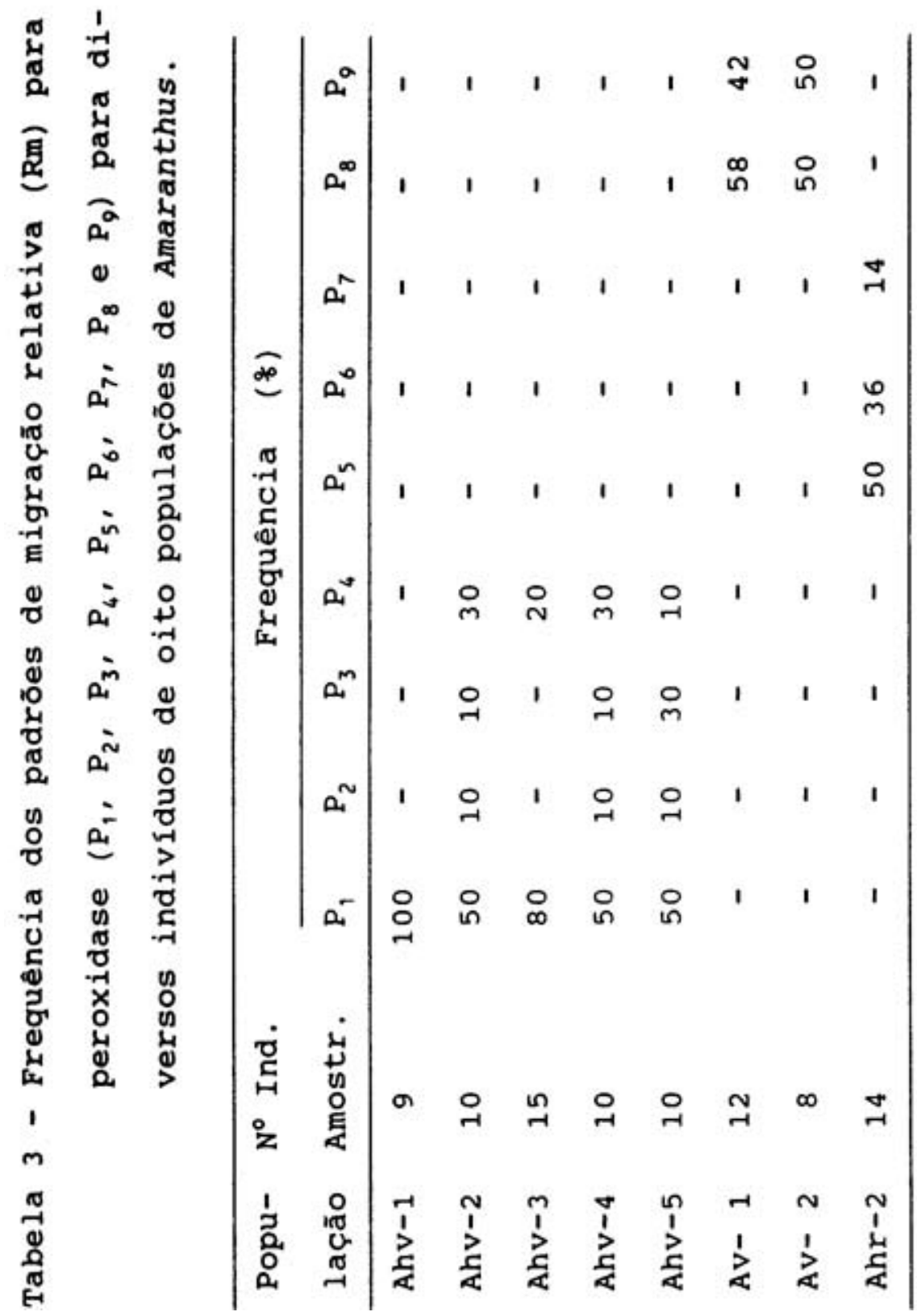


Observa-se pelas Figuras 1 e 2 que as espécies A.hybridus e A.viridis apresentaram padrões izoenzimáticos distintos para esterase e peroxidase, dentro de cada espécie e biótipo.

Para esterase (Figura 1) o único material que apresentou variação foi o A.hybridus tipo verde e em apenas uma banda, ou seja, presença ou ausência da banda na posição 0.7. Por outro lado, para peroxidase, para todas as espécies e biótipos houve variação no padrão de bandas (Figura 2).

Esta resposta foi um pouco diferente da observada por JAIN et alii (1980), onde os autores trabalhando com diversos sistemas enzimáticos, encontraram polimorfismo apenas para os locos da esterase.

Pela Tabela 2, pode-se observar que a variação no padrão de bandas para esterase só ocorreu nas populaçoes Ahv-4 e Ahv-5, de áreas diferentes, porém relativamente próximas. As populaçōes Ahv-1, 2 e 3, provenientes de regiões diferentes mostraram-se monomórficas, com - mesmo padrão de bandas. O A.hybridus tipo roxo, coletado na ESALQ na mesma área de A.Viridis, embora apresente padrão diferente para esterase em relação a esta última, tem duas bandas comuns entre si nas posições 0.17 e 0.65 , sendo que em $A$.viridis a banda na posição 0.65 tem bem maior intensidade (Figura 1). com relação à peroxidase (Tabela 3 ), a única população monomórfica é a de A.hybridus tipo verde coletada na Estação Experimental de Anhembi, Piracicaba. Uniformidade genética em populaçōes de plantas colonizadoras é fato relativamente comum como resultado de efeito de fundação, principalmente no inicio do processo de colonização e reflete a ocorrência de deriva genética. As outras populações mostraram-se polimórficas.

Para peroxidase, as populações de A.hybridus tipo roxo e A.viridis, ambas coletadas na mesma área, na ESALQ, compartili ham as 


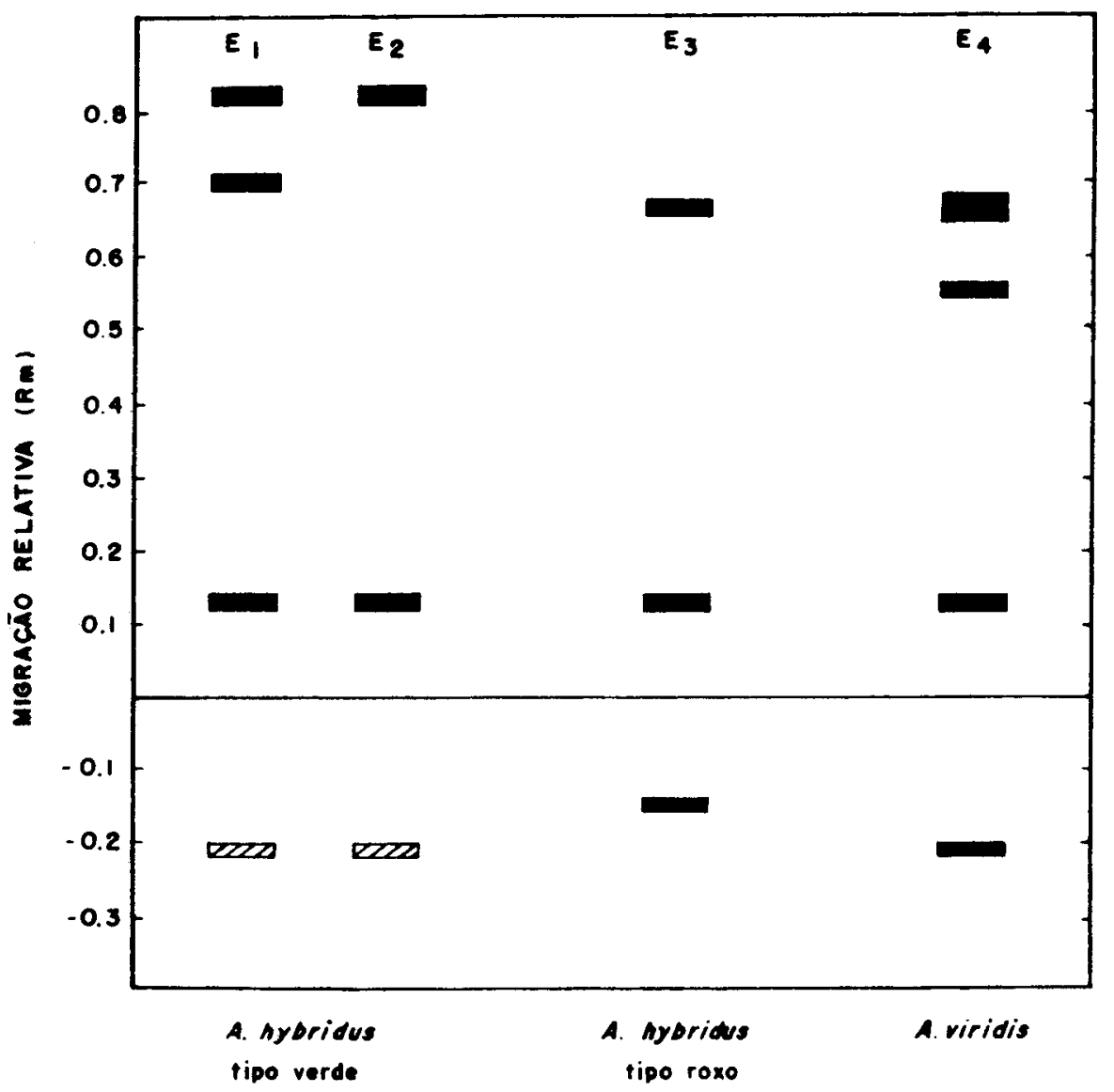

Figura 1 - Padrões de migração (Rm) e intensidades relativas observados para o sistema de esterase catódica e anódica $\left(E_{1}, E_{2}, E_{3}\right.$ e $\left.E_{4}\right)$ em A.hybridus tipo verde e A.hybridus tipo roxo e A.viridis. 


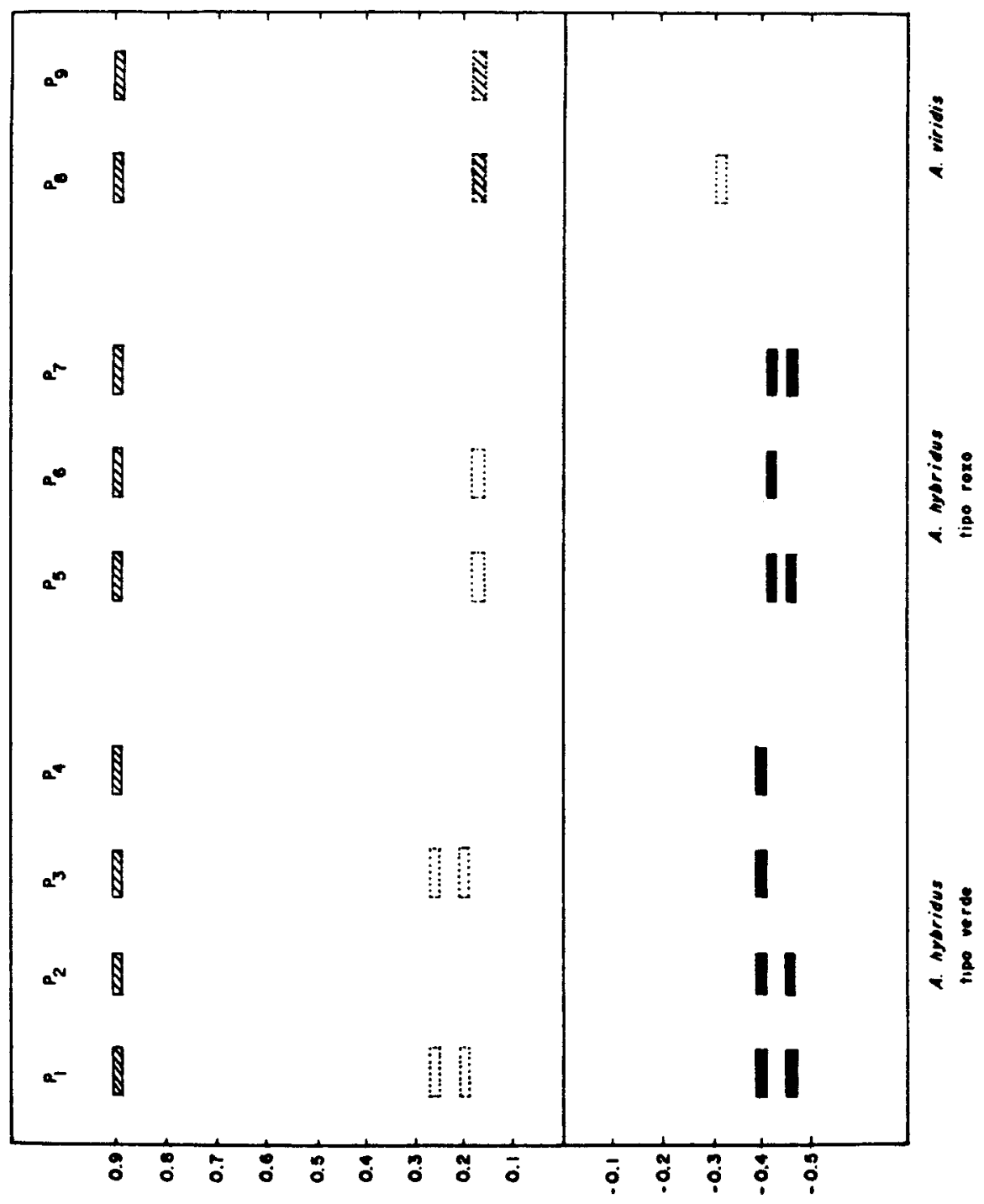

(Wy) VAIเทา3ะ oysvern

Figura 2 - Padróes de migração (Rm) e intensidades relativas observados para 0 sistema de peroxidase catódica e anodica $\left(P_{1}, P_{2}, P_{3}, P_{4}, P_{5}, P_{6}, P_{7}\right.$, $P_{8}$ e $P_{9}$ ) em $A$.hybridus tipo verde $e$ A.hybridus tipo roxo e A.viridis. 
bandas nas posiçōes 0.9 e 0.17 , sendo que para esta última banda as intensidades são diferentes (Figura 2).

E também interessante notar pela Figura 2 que os padrões para peroxidase $\mathrm{P}_{2} e$ A.hybridus tipo verde $\mathrm{e} \mathrm{P}_{2}$ de $A$.hybridus tipo roxo, são semelhantes, pois a pequena diferença de $\mathrm{Rm}$ observada nas bandas anódicas não é suficiente para indicar locos gênicos diferentes.

As respostas obtidas nas análises por eletroforese, apesar de serem restritas devido ao pequeno número de amostras utilizadas e pequeno número de sistemas enzimáticos testados, mostram e existência de variação genética nas espécies e biótipos estudados.

Além disso, apesar das condições experimentais terem sido restritas, o fato das bandas nas posiçōes 0.17 e -0.21 para esterase, serem comuns à A.hybridus tipo verde e A.viridis, além das bandas 0.17 e 0.65 entre A.hybridus tipo roxo e A.viridis, e a banda na posição 0.17 , para peroxidase, ser semelhante em $A$. hybridus tipo roxo e A.viridis, pode indicar um certo grau de hibridação e consequente introgressão entre as espécies. A ocorrência de introgressão em Amaranthus foi observada por COONS (1981) em diversas populações de A.viridis e A.blitum. através da análise de vários caracteres morfológicos.

\section{REFERÊNCIAS BIBLIOGRÁFICAS}

ATKINSON, M.D.; WITHERS, L.A.; SIMPSON, M.J. A. Characterization of cacao germplasm using isozime markers; 1 . A preliminary survey of diversity using starch gel electrophoresis and standardisation of the procedure. Euphytica, Wageningen, 35:741$50,1986$. 
BREWBAKER, J.L.; UPADHYA, M.D. ; MAKINEN, J .; MAC DONALD, T. Isoenzyme polymorphism in flowering plants; III. Gel electrophoretic methods and applications. Physiologia Plantarum, Lund, 21:930-40, 1968.

COLLINS, W.J.; ROSSITER, R.C. ; HAYNES, Y.; BROWN, A.H.D; MARSHALL,D.R. Identification of subterranean clover cultivars and their genetic relationship by isozyme analysis. Australian Journal of Agricultura? Research, Melbourne, 35:399-411, 1984.

COONS, M.P. O gênero Amaranthus em Minas Gerais. Experientiae. Viçosa, 27:1-158, 1981 .

DAMANIA, A.B.; PORCEDUU, E.; JACKSON, M.T. A rapid method for the evaluation of variation in germplasm collections of cereals using polyacrilamyde gel electrophoresis. Euphytica, Wageningen, $\underline{32}: 877-83$, 1983 .

HAUPTLI, H. \& JAIN, S. Genetic structure of landrace populations of the New World grain amaranths. Euphytica, Wageningen, 33: 875-84, 1983 .

HAUPTLI, H. \& JAIN, S. Allozyme variation and evolutionary relationship of grain amaranths (Amaranthus spp). Theoretical and Applied Genetics, Berlin, 69:153-65, 1984 .

HUSSAIN, A.; RAMIREZ, H.; BUSHUK, W. ; ROCA, W. Field bean (Phaseolus vulgaris L.) cultivar indentification by electrophoregrams of cotyledon storage proteins. Euphytica, Wagening, 35: 729-32, 1986. 
HUSSAIN, A.; RAMIREZ, H.; BUSHUK, W.; ROCA, W. Identification of cultivars of forage legume (Desmodium ovalifolium Guill. et Perr.) by their electrophoretic patterns. Canadian Journal of Plant Science, Otawwa, 67:713-7, 1987 .

JAIN, S.K.; WU, L.; VAIDYA, K.R. Levels of morphological an allozyme variation in Indian amaranths: a striking contrast. Journal of Heredity, Washington, 71:283-5, 1980 .

MARCON. G. Estrutura genética de populações de stylosanthes humilis H. B. K. (Leguminosae) de três regiões ecogeográficas do Estado de Pernambuco. Piracicaba, 1988. 179p. (Doutorado - Escola Superior de Agricultura "Luiz de Queiroz"/USP).

MARTINS, P.S. \& JAIN, S.K. Interpopulation variation in rose clover: a recently introduced species in California rangelands. Journal of Heredity, Washington, 71:29-32, 1980 .

RITLAND, $K$. \& JAIN, S. A comparative study on floral and electrophoretic variation with life history variation in Limnanthes alba (Limnanthaceae). Oecologia, Berlin, 64:243-51, 1984 .

SCANDALIOS, J.G. Genetic control of multiple molecular forms of enzymes in plants. Biochemical Genetic, New York, 3:37-39, 1969 .

SCHWAEGERLE, K.E.; GARBUTT, K. ; BAZZAZ, F.A. Differentiation among nine populations of Phlox. 1. Electrophoretic and quantitative variation. Evolution, Lancaster, 40:506$17,1986$. 
ZANGERL, A.R. \& BAZZAZ, F.A. Effects of short-term selection along environmental gradients on variation on population of Amaranthus retroflexus and Abulilon theophrasti. Ecology, Brookling, 65:20717 , $1984 \mathrm{a}$.

ZANGERL, A.R. \& BAZZAZ, F.A. Niche partitioning between two phosphoglucoisomerase genotypes in Amaranthus retroflexus. Ecology, Brookling, 65:218-22, $1984 \mathrm{~b}$.

Trabalho entregue para publicação em 06.11.89 Trabalho aprovado para publicação em 25.02.91 